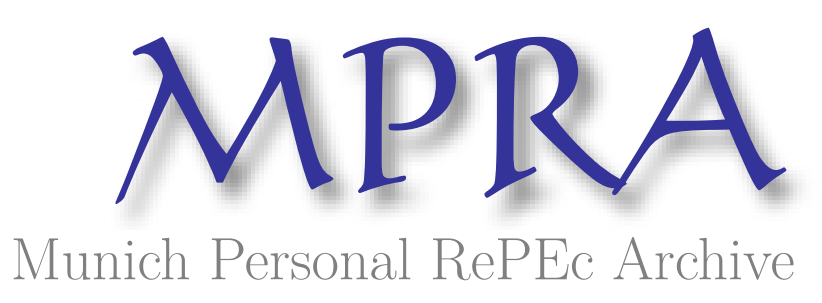

\title{
Sequentially Rationalizable Choice with Transitive Rationales
}

Au, Pak Hung and Kawai, Keiichi

2011

Online at https://mpra.ub.uni-muenchen.de/29687/

MPRA Paper No. 29687, posted 19 Mar 2011 19:16 UTC 


\title{
Sequentially Rationalizable Choice with Transitive Rationales
}

\author{
Pak Hung Au*and Keiichi Kawai ${ }^{\ddagger}$
}

January 17, 2011

\begin{abstract}
In this note, we analyze a sequentially rationalizable choice model with a transitive rationale and a standard preference. The model in this note is more restrictive than the Rational Shortlist Method (RSM) model which is proposed in Manzini and Mariotti (2007) to capture cyclic behavior. Yet, a decision maker in our model exhibits cyclic behavior in general. We prove that the cyclicity of an indirectly revealed preference is exactly what distinguishes the RSM framework (a sequential choice model with a non-standard preference) from our transitive-RSM framework (a sequential choice model with a standard preference). We also provide a partial identification result on the representation.
\end{abstract}

JEL Codes: D01.

Keywords: Sequentially rationalizable choice, Rationalization.

There is a growing literature that models behavioral anomalies resulting from internal conflict. In a seminal paper, Manzini and Mariotti (2007) propose a sequentially

*Department of Economics, Northwestern University; p-au@northwestern.edu.

${ }^{\dagger}$ Department of Economics, Northwestern University; keiichi-kawai@northwestern.edu.

${ }^{\ddagger}$ We would like to thank Eddie Dekel and an anonymous referee for comments and suggestions that greatly improved this note. We acknowledge financial support from the Center for Economic Theory of the Economics Department of Northwestern University. 
rationalizable choice model that accommodates cyclic choice behavior, which is common anomaly observed in experiments. ${ }^{1)}$ The objective of this note is to analyze the gap that exists between standard choice theory and the sequential choice model in Manzini and Mariotti (2007), which they term the rational shortlist method (RSM) model.

A decision maker (DM) in the RSM framework has two rationales. She departs from the standard theory in the following respects: (i) the DM uses her first rationale to form a shortlist, from which she makes her final choice using the second rationale; (ii.a) the DM's first rationale may be intransitive; and (ii.b) the DM's second rationale may be cyclic. ${ }^{2)}$ The RSM model encompasses at least two interpretations: a) the first rationale can be interpreted as the DM's (potentially incomplete) underlying strict preference, and the second as the "tie-breaking rule," and b) the first rationale can be interpreted as some selection criterion that the DM deems important; and the second rationale as the DM's underlying preference. Hence, an RSM choice function is observationally equivalent to the standard choice function if the first rationale is a complete strict preference (under interpretation a)), or if the first rationale is empty and the second rationale is a complete strict preference (under interpretation b)). Whichever interpretation is employed, a non-standard preference is permitted in the RSM framework. Therefore, behavioral anomalies that are identified in the RSM framework may be the result of the procedure by which a shortlist is created before the final decision is made, or a non-standard preference, or a combination of the two.

In this note, we employ interpretation b). In our model, which we call a transitiveRSM model, while (i) the DM still forms a shortlist before making the final choice, (ii.a') her first rationale (her criterion) is transitive, and (ii.b') her second rationale (her preference) is complete and transitive. Therefore, our model departs from the standard choice model only if the DM's criterion is neither empty nor complete. Since a shortlist

\footnotetext{
${ }^{1)}$ For example, in the experiment conducted by Loomes et al. (1991), out of 200 subjects, 128 subjects exhibited some form of cyclic choice. Also see Tversky (1972) and Starmer and Sugden (1998).

${ }^{2}$ In general, there are multiple pairs of rationales that sequentially rationalize an RSM choice function. However, some RSM choice functions can be sequentially rationalized only by a cyclic second rationale.
} 
is formed if and only if the criterion is nonempty, any behavioral anomalies in our model must result from the presumption that the DM forms a shortlist.

Conditions (ii.a') and (ii.b') are imposed in order to keep the departure of our model from the standard theory minimal. We find these are reasonable assumptions. The reason for imposing condition (ii.a') is the following. Transitivity of the criterion requires that if $y$ beats $x$, then $x$ can never matter to any set containing $y$. In other words, the shortlists from $S$ and $S \backslash\{x\}$ have to be identical whenever an alternative $x$ is not in the shortlist from a choice set $S$ (that contains $x$ and $y$ ). Condition (ii.b') is equivalent to requiring that the underlying preference is a standard (strict) preference. ${ }^{3)}$

Our representation theorem (Theorem 1) shows that an RSM model is behaviorally different from a transitive-RSM model if and only if one axiom, which we call the axiom of No Binary Chain Cycles (NBCC), is violated. Our second result (Theorem 2) identifies the set of criterion-preference pairs that rationalize a given choice function. We then identify the condition on the choice function under which the criterion-preference representation is unique (Corollary 1 ).

Our note is closely related to Cherepanov et al. (2008). Like our note, the DM in their paper forms a shortlist, and then makes a choice according to a standard preference over alternatives. Unlike our note, however, the DM has multiple criteria, and the shortlist consists of all alternatives that are maximal with respect to at least one of the criteria. They introduce an axiom which imposes acyclicity on the revealed preference, and then show that the axiom completely characterizes choice behavior that arises from their model. The notion of the revealed preference in their paper is similar to but independent from ours. As a result, their axiom is neither weaker nor stronger than our NBCC axiom. Nevertheless, it can be easily verified that their axiom is implied by our NBCC axiom and the Weak WARP introduced in Manzini and Mariotti (2007).

\footnotetext{
3) Another reason for studying a restrictive sequential choice model is that if we do not get a unique representation, or comparative static result in this most restrictive class, we never get them in a more general model.
} 
Other related papers include Aspesteguia and Ballester (2009), García-Sanz and Alcantud (2010), and Manzini and Mariotti (2010). In contrast to our note, all these works generalize the original model in Manzini and Mariotti (2007).

Aspesteguia and Ballester (2009) consider a sequential choice model with acyclic rationales. They axiomatize the choice behavior of a DM who employs a sequence of incomplete and acyclic rationales to eliminate alternatives from the choice set until she reaches a unique element, which is her final choice. Their axiom is about the existence of a binary selector (a function that selects a binary choice problem from an arbitrary choice problem) that satisfies a consistency requirement. In contrast, our set of axioms is directly imposed on the choice function, and therefore is easily comparable with axioms in other papers.

Manzini and Mariotti (2010) analyze a choice model in which a choice is induced by the sequential application of semiorders. ${ }^{4)}$ They show that the model restricts choice data the same way as the sequential choice model with an arbitrary number of acyclic rationales on any finite domain. The transitive-RSM model proposed here can thus be viewed as a special case of their model when the domain is finite. Finally, García-Sanz and Alcantud (2010) characterize choice correspondences that can be rationalized by the sequential application of two relations.

\section{Basic Definitions}

Consider an arbitrary non-empty finite set $X$ with $|X| \geq 3$. The set of choice problems is $\Omega_{X} \equiv 2^{X} \backslash\{\emptyset\}$. A choice function on $X$ is defined as any function $c: \Omega_{X} \rightarrow X$ with $c(S) \in S$ for all $S \in \Omega_{X}$. The decision maker (DM) has an asymmetric, complete, and transitive (strong) preference relation $P_{2} \in X \times X$. We denote the $P_{2}$-maximum element of $S$ by $c_{2}\left(S ; P_{2}\right)$. A criterion is an asymmetric and transitive binary relation, $P_{1} \subset X \times X$. We call the set of $P_{1}$-maximal elements of $S, \max \left(S ; P_{1}\right) \equiv\left\{x \in S: \nexists y\right.$ s.th. $\left.y P_{1} x\right\}$, the shortlist

\footnotetext{
${ }^{4)}$ A semiorder is a transitive binary relation. See Luce (1956) for the definition.
} 
from $S$. For notational convenience, we denote $c_{2}\left(\max \left(S ; P_{1}\right) ; P_{2}\right)$ by $c_{\left(P_{1}, P_{2}\right)}(S)$.

Definition 1 A choice function cis a transitive rational shortlist method (transitive-RSM) if there exists a pair of criterion and preference $\left(P_{1}, P_{2}\right)$ such that $c(S)=c_{\left(P_{1}, P_{2}\right)}(S)$ for all $S \in \Omega_{X}$.

The DM in our model makes a choice using the following procedure. First, she uses the criterion $P_{1}$ to eliminate all inferior alternatives and get a shortlist. (Formally, she eliminates $y \in S$ if and only if there exists an $x \in S$ such that $x P_{1} y$ ). Then she picks an alternative from the shortlist using her preference $P_{2}$.

To facilitate discussion, the following definitions and results from Manzini and Mariotti (2007) are restated. In Manzini and Mariotti (2007), an asymmetric binary relation on $X \times X$ is called a rationale.

Definition 2 (Manzini and Mariotti (2007)) A choice function $c$ is a rational shortlist method $(R S M)$ if there exist two rationales $P_{1}$ and $P_{2}$ such that $c(S)=c_{2}\left(\max \left(S ; P_{1}\right) ; P_{2}\right)$. The pair $\left(P_{1}, P_{2}\right)$ is said to sequentially rationalize $c$.

Manzini and Mariotti (2007) show that a choice function $c$ is an RSM if and only if $c$ satisfies the following two axioms:

Weak WARP Suppose $x=c(\{x, y\})$, and $x=c(S)$ for some $S \ni x, y$. Then for any $T$ such that $\{x, y\} \subset T \subset S, c(T) \neq y$.

Expansion If $x=c(S)=c(T)$, then $x=c(S \cup T)$.

While a transitive-RSM requires transitivity for both $P_{1}$ and $P_{2}$ (and completeness for $P_{2}$ ), an RSM requires transitivity for neither $P_{1}$ nor $P_{2}$. Therefore, a transitive-RSM choice function is an RSM choice function, but not vice versa. Nevertheless, a transitive-RSM choice function permits cyclic choice behavior, as shown by the following example.

Example 1 Suppose $X=\{x, y, z\}, c(X)=x, c(\{x, y\})=x, c(\{y, z\})=y$, and $c(\{x, z\})=z$. Then the following pair of $\left(P_{1}, P_{2}\right)$ rationalizes the choice function $c: P_{1}=\{(y, z)\}$ and $P_{2}=$ $\{(z, x),(x, y),(z, y)\}$. Therefore $c$ is a transitive-RSM choice function. 


\subsection{Revealed Preference}

Suppose a DM in our model chooses $x$ over $y$ from $\{x, y\}$, i.e. $x=c(\{x, y\})$. There are three possible explanations for this choice: (i) $x$ ranks above $y$ in her criterion $P_{1}$ and $x$ is preferred to $y$ according to $P_{2}$; (ii) $x$ ranks above $y$ in her criterion $P_{1}$ but $y$ is preferred to $x$ according to $P_{2}$; and (iii) $x$ neither dominates or is dominated by $y$ in $P_{1}$, but $x$ is preferred to $y$ in $P_{2}$.

If (i) or (ii) is the reason for $x=c(\{x, y\})$, then the DM's shortlists from choice sets $S \ni x, y$ and $S \backslash\{y\}$ coincide. Therefore, $S$ and $S \backslash\{y\}$ induce the same choice. On the other hand, if (iii) is the explanation for $x=c(\{x, y\})$, then the DM's shortlists from $S \ni x, y$ and $S \backslash\{y\}$ may be different. As a result, the choices from $S$ and $S \backslash\{y\}$ may be different. Hence, if we observe $c(S) \neq c(S \backslash\{y\})$ for some $S \ni x, y$, we may conclude that the alternative $x$ is revealed as being preferred to $y$. The above discussion yields the following definitions. ${ }^{5)}$

Definition 3 We say $x$ is directly revealed preferred to $y$, which is denoted as $x>_{D}^{c} y$, if and only if $c(\{x, y\})=x$ but $c(S) \neq c(S \backslash\{y\})$ for some $S \ni x, y$.

Definition 4 A set $S=\left\{x_{1}, \ldots, x_{n}\right\}$ is called a binary chain when $x_{k}>_{D}^{c} x_{k+1}$ for all $k \leq n-1$

Definition 5 We say $x_{1}$ is indirectly revealed preferred to $x_{n}$, which is denoted as $x_{1}>_{I}^{c} x_{n}$, if and only if there exists $\left\{x_{2}, \ldots, x_{n-1}\right\}$ such that $\left\{x_{1}, \ldots, x_{n}\right\}$ is a binary chain.

Suppose $\left\{x_{1}, \ldots, x_{n}, x_{1}\right\}$ is a binary chain. Then $x_{1}$ is indirectly revealed preferred to $x_{1}$.

\footnotetext{
${ }^{5)}$ Our notion of the revealed preference is similar to the one in Masatlioglu et al. (2010). They consider a choice model in which the DM is characterized by two unobservables: (i) her underlying preference, and (ii) an attention filter which reduces every choice set to a subset called a consideration set to which preferences are applied. The DM may select an alternative $x$ in the presence of $y$ even when the DM prefers $y$ because $y$ is not in the consideration set. In their framework, the underlying preference and the attention filter can be recovered if the following choice behavior is observed: $c(S)=x$ for some $S \ni x, y$ but $c(S \backslash\{y\}) \neq c(S)$. This can happen only when her consideration set has changed, i.e., $y$ must have been considered in $S$ (revealed attention). Also, the fact that $x$ is chosen while $y$ is also in the consideration set implies that $x$ should be revealed preferred to $y$ (revealed preference).
} 
In other words, some cyclic choices can only be explained by cyclic preference relations. To minimize the departure from the standard model, we impose the following axiom. ${ }^{6)}$ NBCC A choice function $c$ satisfies No Binary Chain Cycles Axiom (NBCC) if there does not exist any binary chain $\left\{x_{1}, \ldots, x_{n}\right\}$ such that $x_{1}=x_{n}$.

Note that NBCC does NOT rule out cyclic choice behavior. It only rules out choice functions that reveal a cyclic preference relation. As the following example shows, It is violated by some RSM. As we shall show below, NBCC is the axiom that distinguishes a transitive-RSM choice function from an RSM choice function.

Example 2 Suppose $X=\{w, x, y, z\}$; and $c^{-1}(w)=\{\{w, x\},\{w, x, y\}\}, c^{-1}(x)=\{\{x, y\},\{x, y, z\}, X\}$, $c^{-1}(y)=\{\{y, z\},\{w, y\},\{w, y, z\}\}$, and $c^{-1}(z)=\{\{x, z\},\{w, z\},\{w, x, z\}\}$. Then $c$ is an RSM choice function. ${ }^{7)}$ We have $x>_{D}^{c}$ y because $x=c(\{x, y\})$ and $c(\{x, y, z\})=x \neq z=c(\{x, z\})$. Similarly, $w>_{D}^{c} x$ because $w=c(\{w, x\})$ and $c(\{w, x, y\})=w \neq y=c(\{w, y\})$. Also, $y>_{D}^{c} w$ because $y=c(\{w, y\})$ and $c(\{w, x, y\})=w \neq x=c(\{x, y\})$. Therefore, the directly revealed preference exhibits a cycle, thus violating NBCC.

\section{Main Results}

Now we are ready to state our first main result:

Theorem 1 An RSM choice function $c$ is a transitive-RSM choice function if and only if $c$ satisfies NBCC.

Proof. In Appendix.

The "if" part of the proof to 1 is constructive. Given an RSM choice function $c$ that satisfies NBCC, we define $P_{2}^{c}$ as a completion of $>_{1}^{c}$ and $P_{1}^{c}$ as follows:

$$
P_{1}^{c} \equiv\{(x, y): c(S)=c(S \backslash\{y\}) \text { for any } S \ni x, y \text { with } x \neq y\} .
$$

${ }^{6)}$ We think that the axiom is natural because under our interpretation, the second rationale is a strict preference relation. It is therefore reasonable to impose acyclicity on it.

${ }^{7)} \mathrm{C}$ is sequentially rationalized by $P_{1}=\{(x, y),(y, z),(z, w)\}$ and $P_{2}=\{(y, w),(w, x),(z, x)\}$. 
Then, it is not difficult to see that both $P_{1}^{c}$ and $P_{2}^{c}$ are asymmetric and transitive. The key part of the proof is to show that the pair $\left(P_{1}^{c}, P_{2}^{c}\right)$ represents the choice function $c$, i.e., $c_{\left(P_{1}^{c}, P_{2}^{c}\right)}(S)=c(S)$ for all $S$.

Remark 1 It can be shown that $P_{1}^{c}$ in (2.1) satisfies $P_{1}^{c}=\{(x, y): c(\{w, x, y, z\})=c(\{w, x, z\})$ for any $w, z \in X, A N D x \neq y\}$. Therefore, the criterion can be constructed by observing all choice behavior from choice sets of size 4 or less. Recall that in Manzini and Mariotti (2007), the first rationale was constructed as follows: $x P_{1}^{c} y$ if and only if $y \neq c(S)$ for all $S \ni x, y$. Therefore, we need much less information to construct $P_{1}^{c}$.

Next, we discuss the identification problem of our representation. A few definitions are required. For a transitive-RSM choice function $c$, we denote $P_{1}^{c}$ defined in (2.1) by $\bar{P}_{1}^{c}$. In addition, define $\mathcal{P}(c) \equiv\left\{\left(P_{1}, P_{2}\right): c_{\left(P_{1}, P_{2}\right)}(S)=c(S)\right\}, \mathcal{P}_{i}(c) \equiv\left\{P_{i}:\left(P_{1}, P_{2}\right) \in \mathcal{P}(c)\right.$ for some $\left.P_{j} \neq P_{i}\right\}$, and $\mathcal{P}_{2}\left(P_{1} ; c\right) \equiv\left\{P_{2}:\left(P_{1}, P_{2}\right) \in \mathcal{P}(c)\right\}$.

Theorem 2 Let $c$ be a transitive-RSM choice function. Define $\underline{P}_{1}^{c} \equiv\left\{(x, y) \in \bar{P}_{1}^{c}: y>_{I}^{c} x\right\}$. We have (i) $\underline{P}_{1}^{c}, \bar{P}_{1}^{c} \in \mathcal{P}_{1}(c)$; (ii) for all $P_{1}^{c} \in \mathcal{P}_{1}$ (c), (ii.a) $\underline{P}_{1}^{c} \subset P_{1}^{c} \subset \bar{P}_{1}^{c}$, and (ii.b) $\mathcal{P}_{2}\left(\underline{P}_{1}^{c} ; c\right) \subset$ $\mathcal{P}_{2}\left(P_{1}^{c}, c\right) \subset \mathcal{P}_{2}\left(\bar{P}_{1}^{c}, c\right)$ and $\left|\mathcal{P}_{2}\left(\underline{P}_{1}^{c} ; c\right)\right|=1$.

Proof. In Appendix.

Parts (i) and (ii.a) of Theorem 2 identify the tight upper and lower bounds for the criterion $P_{1}^{c}$. The upper bound tells us when a particular alternative makes some other alternatives irrelevant to the choice. To be precise, whenever $c(S)=c(S \backslash\{y\})$ for all $S \ni x, y$, we have $x \bar{P}_{1}^{c} y$.

The lower bound captures the "conflict" between the upper bound and the indirectly revealed preference. ${ }^{8)}$ Even if $y$ is irrelevant to the final choice whenever $x$ is in the choice set (i.e., $x \bar{P}_{1}^{c} y$ ), $y$ may be indirectly revealed preferred to $x$ (i.e., $y>_{I}^{c} x$ ). Whenever this is the case, we have $x \underline{P}_{1}^{c} y$.

Part (ii.b) says that a less complete $P_{1}^{c}$ imposes more restrictions on $P_{2}^{c}$ in the task of rationalizing the choice function. In particular, when the tight lower bound $\underline{P}_{1}^{c}$ is used,

${ }^{8)}$ When a choice function $c$ satisfies WARP, $\underline{P}_{1}^{c}=\emptyset$, while $\bar{P}_{1}^{c}$ is a linear order. 
there is a unique preference that rationalizes the choice function. An immediate corollary of Theorem 2 is the following.

Corollary 1 The preference is point-identified if and only if the criterion is point-identified, i.e., $\left|\mathcal{P}_{2}(c)\right|=1$ if and only if $\underline{P}_{1}^{c}=\bar{P}_{1}^{c}$. Therefore, a transitive-RSM choice function $c$ has a unique criterion-preference representation if and only if $c(S)=c(S \backslash\{y\})$ for all $S \ni x, y$ implies $y>_{I}^{c} x$.

Remark 2 (Behavioral Comparative) Suppose there are two transitive-RSM choice functions, $c^{i}$ and $c^{j}$, and they are uniquely represented by $\left(P_{1}^{c^{i}}, P_{2}^{c^{i}}\right)$ and $\left(P_{1}^{c^{j}}, P_{2}^{c^{j}}\right)$ respectively. Suppose further that $P_{1}^{c^{i}} \subset P_{1}^{c^{j}}$ and $P_{2}^{c^{i}}=P_{2}^{c^{j}}$. In other words, $c^{j}$ exhibits more "conflicts" between the criterion and the preference. Given that an RSM choice function violates WARP if and only if it exhibits cycles (Theorem 2 in Manzini and Mariotti (2007)), one may expect $c^{j}$ to exhibit "more cyclic behaviors" than $c^{i}$. However, as the following example shows, this conjecture turns out to be false.

Example 3 Suppose that $X=\{w, x, y, z\}$ and $D M-i$ and $D M-j$ have the following pair of criteria and preferences: $P_{1}^{c^{i}}: x P_{1}^{i} z, y P_{1}^{i} w, y P_{1}^{i} z ; P_{2}^{c^{i}}: z P_{2}^{i} w P_{2}^{i} x P_{2}^{i} y ; P_{1}^{c^{j}}: x P_{1}^{j} z, y P_{1}^{j} w$; and $P_{2}^{c^{j}}: z P_{2}^{i} w P_{2}^{i} x P_{2}^{i} y$. Consider $c^{i}=c_{\left(P_{1}^{c^{i}}, P_{2}^{c^{j}}\right)}$, and $c^{j}=c_{\left(P_{1}^{c^{j}}, P_{2}^{c^{j}}\right)}$. Then $\underline{P}_{1}^{c^{i}}=\bar{P}_{1}^{c^{i}} \supseteq \underline{P}_{1}^{c^{j}}=\bar{P}_{1}^{c^{j}}$, and $P_{2}^{c^{i}}=P_{2}^{c^{j}}$. Nevertheless, both $c^{i}$ and $c^{j}$ exhibit two cycles of length 3 , and one cycle of length 4. Moreover, the cycles of length 4 by $c^{i}$ and $c^{j}$ are different.

\section{Conclusion}

The contribution of this note is twofold. First, we show that even with a standard preference, the DM in general exhibits cyclic behavior if she uses the shortlist method to make her decision. We prove that the cyclicity of indirectly revealed preference is exactly what distinguishes the RSM framework (a sequential choice model with a nonstandard preference), from the transitive-RSM framework (a sequential choice model with a standard preference) that is proposed here. 
Second, we provide a partial identification result on the representation. In general, there are multiple pairs of criteria and preferences that rationalize a transitive-RSM choice function. Our partial identification result provides the tight bounds of the criterion and the preference representations, as well as the necessary and sufficient condition on the choice function for a unique criterion-preference representation.

\section{Appendix}

\subsection{Proof of Theorem 1}

We say that a choice function $c$ satisfies Weak-IIA if the following condition is met.

Weak-IIA Let $\{x, y\} \subset T \subset S$. If $x=c(S)$ and $y=c(T)$, then there exists a $z \in S \backslash T$ such that $c(U)=c(U \backslash\{y\})$ for all $\left.U \ni y, z .{ }^{9}\right)$

As an intermediate step, we prove the following lemma. By following lemma, Theorem 1 follows if we show that a choice function $c$ is a transitive-RSM if and only if $c$ satisfies Weak-IIA and NBCC.

Lemma 1 Suppose a choice function c satisfies NBCC. Then $c$ satisfies Weak-IIA if and only if $c$ is an RSM.

Proof. (The "only if" part) We show that Weak-IIA implies Weak WARP. Suppose $x=c(\{x, y\})=c(S)$ for $S \ni x, y$, and $c(T)=y$ for some $T$ such that $\{x, y\} \subset T \subset S$. Given that $x=c(\{x, y\})$ and $y=c(T)$, there exists a $z \in T \backslash\{x, y\}$ such that $c(U)=c(U \backslash\{x\})$ for all $U \ni x, z$. This contradicts $c(S)=x$, because $x, z \in S$.

\footnotetext{
${ }^{9}$ Weak-IIA says the following. Suppose $x$ and $y$ are available in two choice sets $S$ and $T$, where $T \subset S$. In addition, suppose the DM chooses $x$ from $S$, but switches to $y$ when she faces $T$. Then in a sequential choice framework, a natural interpretation is that there is an alternative $z$ which is in $S$ but not $T$, and such a $z$ leads the DM not to consider $y$. When the DM faces $T$, such a $z$ is not present, and therefore she considers $y$. If this is the case, the alternative $y$ should be irrelevant to the DM whenever $z$ is available.
} 
Next, we show that Weak-IIA implies Expansion. Assume that $x=c(S)=c(T)$ but $y=c(S \cup T) \neq x$, by contradiction. Then by Weak-IIA, and $x=c(S) \neq c(S \cup T)=y$, we know that there exists a $z \in T$ such that $c(U)=c(U \backslash\{x\})$ for all $U \ni x, z$. This is a contradiction because $x, z \in T$ and therefore $c(T)=x \neq c(T \backslash\{x\})$.

(The "if" part) To show the "if" part, we define the following notion first. A choice function $c$ is said to satisfy Reduction Axiom if the following property is satisfied: when $x=c(S)$ and $y=c(T)$ for $S$ and $T$ such that $\{x, y\} \subset T \subset S$ : there exists a $z \in S \backslash T$ such that $c(\{x, y, z\})=x, c(\{x, z\})=x, c(\{y, z\})=z$, and $c(\{x, y\})=y$.

Claim 1 Weak WARP, Expansion, and NBCC together imply Reduction.

Proof. Suppose there are S and T such that $x=c(S), y=c(T)$ and $\{x, y\} \subset T \subset S$. We first define the following sets: $T^{\prime} \equiv T \cup\{z \in S: c(\{y, z\})=y\}, S^{\prime} \equiv T^{\prime} \cup\{z \in S: c(\{x, z\})=z$ or $c(\{x, y, z\})=x\}$. Note that $T \subset T^{\prime} \subset S^{\prime} \subset S$ by construction. Furthermore, define $Z \equiv S^{\prime} \backslash T^{\prime}$ and $Z^{\prime} \equiv$ $\{z \in Z: c(\{x, z\})=x\} \subset Z$.

We make the following observations: (i) $c(\{x, y\})=y$ and $y>_{D}^{c} x$; (ii) $y=c\left(T^{\prime}\right)$; and (iii) $x=c\left(S^{\prime}\right)$.

To see (i), note that $x=c(S)$ and $y=c(T)$ together with Weak WARP imply $c(\{x, y\})=y$. Then $c(S)=x$ implies $y>_{D}^{c} x$. To see (ii), note that $y=c(T)$, and $c(\{y, z\})=y$ for all $z \in T^{\prime} \backslash T$. Therefore, $y=c\left(T^{\prime}\right)$ follows from Expansion. To see (iii), fix any $z \in S \backslash S^{\prime}$, we have $c(\{y, z\})=z, c(\{x, z\})=x$ and $c(\{x, y, z\}) \neq x$. By Weak WARP, $c(\{x, y, z\}) \neq z$ because $c(\{x, z\})=x$ and $c(S)=x$. Therefore, we have $c(\{x, y, z\})=y$. Moreover, $c(\{y, z\})=z$ and $c(\{x, z\}) \neq c(\{x, y, z\})$ implies that $z>_{D}^{c} y$. Observation (i) and NBCC imply $\neg\left(x>_{D}^{c} z\right)$. However, we have $c(\{x, z\})=x$. Therefore, for all $U \ni x, z$, we have $c(U)=c(U \backslash z)$. This gives $x=c\left(S^{\prime}\right)$.

Take any $z \in Z^{\prime}$. By the definition of $Z^{\prime}$, we have $c(\{x, z\})=x$ and $c(\{x, y, z\})=x$. By definitions of $T^{\prime}$, and $Z \prime$, we have $c(\{y, z\})=z$. By observation $(i)$, we have $c(\{x, y\})=y$. Therefore, the proof is complete if we can show that $Z^{\prime}$ is non-empty.

Suppose $Z^{\prime}=\emptyset$. Given that $c$ satisfies Weak WARP and Expansion, $c$ is sequentially 
rationalizable. Let $\left(P_{1}^{c}, P_{2}^{c}\right)$ be a pair of rationales that sequentially rationalizes the choice. ${ }^{10)}$ For all $z \in Z, c(\{x, z\})=z$ implies that either $z P_{1}^{c} x$ or $z P_{2}^{c} x$. Similarly, for all $z \in Z, c(\{y, z\})=z$ implies that either $z P_{1}^{c} y$ or $z P_{2}^{c} y$. Therefore, we have $c\left(\{x, y\} \cup Z^{\prime \prime}\right) \neq x, y$ for all $Z^{\prime \prime} \subset Z$.

Let $Z_{0}=Z$, and $Z_{n+1}=Z_{n} \backslash\left\{z_{n}\right\}$, where $z_{n}=c\left(\{x, y\} \cup Z_{n}\right)$. Note that $z_{n} \neq x, y$ for all $n$. Then observation (iii) implies that there is a $w \in T^{\prime}$ such that $w P_{1}^{c} z_{0}$. Therefore, $c\left(T^{\prime} \cup\left\{z_{0}\right\}\right)=$ $c\left(T^{\prime}\right)$. Define $T_{n+1}=T_{n} \cup\left\{z_{n}\right\}$ with $T_{0}=T^{\prime}$. Then a similar argument yields $c\left(T_{n+1}\right)=$ $c\left(T_{n}\right)=c\left(T^{\prime}\right)$. Given that $S^{\prime}$ is finite, we have $c\left(S^{\prime}\right)=c\left(T_{|\mathrm{Z}|-1}\right)=c\left(T^{\prime}\right)$. This contradicts with observations (ii) and (iii) above.

Claim 2 Reduction and NBCC imply Weak-IIA.

Proof. Suppose there is a set $x=c(S)$ and $y=c(T)$ for some $S, T$ such that $\{x, y\} \subset T \subset S$. Note that by Reduction, there exists a $z \in S \backslash T$ such that $c(\{x, y, z\})=x, c(\{x, z\})=x, c(\{y, z\})=z$, and $c(\{x, y\})=y$. Hence, we have $y>_{D}^{c} x>_{D}^{c} z$. If there exists a $U$ such that $c(U) \neq c(U \backslash\{y\})$ and $y, z \in U$, then $z>_{D}^{c} y$, which violates NBCC.

By Lemma 1, we are done if we show that a choice function $c$ is a transitive-RSM if and only if $c$ satisfies Weak-IIA and NBCC.

(The "if" part) The proof is constructive. The criterion is the following: let $P_{1}^{c} \equiv$ $\{(x, y): c(S)=c(S \backslash\{y\})$ for all $S \ni x, y$ and $x \neq y\}$. Next, we define $P_{2}^{c}$ as a completion of $>_{I}^{c}$. Note that by Szpilrajn's Theorem, such a completion exists. Below, we show that both $P_{1}^{c}$ and $P_{2}^{c}$ are asymmetric and transitive. Then, we show that $c_{\left(P_{1}^{c}, P_{2}^{c}\right)}(S)=c(S)$.

First, we argue that the binary relation $P_{1}^{c}$ is asymmetric and transitive. That $P_{1}^{c}$ is asymmetric follows from the definition of $P_{1}^{c}$ together with the single valuedness of $c$ and the assumption of full domain. To see that $P_{1}^{c}$ is transitive, assume $x P_{1}^{c} y$ and $y P_{1}^{c} z$. The proof is complete if we show $c(S)=(S \backslash\{z\})$ for all $S \ni x, z$. Take an arbitrary set

\footnotetext{
${ }^{10)}$ The existence of such $\left(P_{1}^{c}, P_{2}^{c}\right)$ is guaranteed because $c$ satisfies Weak WARP and Expansion (Theorem 1 in Manzini and Mariotti (2007)).
} 
$S \ni x, z$. First, suppose that $y \in S$. Then by $y P_{1}^{c} z$, we have $c(S)=c(S \backslash\{z\})$. Next, suppose $y \notin S$. We know that $c(S \cup\{y\} \backslash\{z\})=c(S \cup\{y\})$ by $y P_{1}^{c} z$, and $c(S \cup\{y\})=c(S)$ by $x P_{1}^{c} y$. In addition, $x P_{1}^{c} y$ implies $c(S \cup\{y\} \backslash\{z\})=c(S \backslash\{z\})$. Hence, we have $c(S)=c(S \backslash\{z\})$.

Next, we show that the binary relation $P_{2}^{c}$ is asymmetric and transitive. This is straightforward due to the fact that $>_{I}^{c}$ is asymmetric by NBCC, so is $P_{2}^{c}$.

Lastly, we show $c(S)=c_{\left(P_{1}^{c}, P_{2}^{c}\right)}(S)$ for all $S$. Suppose $x=c(S)$. First, we show that $x \in \max \left(S ; P_{1}^{c}\right)$. If not, then there is a $y$ such that $y P_{1}^{c} x$, and therefore $c(S)=c(S \backslash\{x\})$, which is a contradiction. Next, we show that $x>_{I}^{c} y$ for all $y \in \max \left(S ; P_{1}^{c}\right)$. If not, then for some $y \in \max \left(S ; P_{1}^{c}\right)$, we have $y=c(\{x, y\})$. Then weak-IIA implies that $z \in S \backslash\{x, y\}$ such that $z P_{1}^{c} y$, which contradicts $y \in \max \left(S ; P_{1}^{c}\right)$.

(The "only if" part) Suppose $c$ is a transitive-RSM, and $c(S)=c_{\left(P_{1}^{c}, p_{2}^{c}\right)}(S)$ for some $\left(P_{1}^{c}, P_{2}^{c}\right)$. If $x>_{D}^{c} y$, then $x P_{2}^{c} y$. Therefore, the completeness and the transitivity of $P_{2}^{c}$ imply NBCC. Next, suppose $x=c(S)$ and $y=c(T)$ for $\{x, y\} \subset T \subset S$. It is straightforward to check $x \in \max \left(S ; P_{1}^{c}\right), y \notin \max \left(S ; P_{1}^{c}\right)$, and $x, y \in c\left(T ; P_{1}^{c}\right)$.

Therefore, there exists $z \in S \backslash T$ such that $z P_{1}^{c} y$ and $x P_{2}^{c} z$. By transitivity of $P_{1}^{c}$, we have $z P_{1}^{c} w$ for all $w$ such that $y P_{1}^{c} w$. Hence, $c(U)=c(U \backslash\{y\})$ for all $U \ni y$, $z$. Q.E.D.

\subsection{Proof of Theorem 2}

Proof of (i): $\quad \bar{P}_{1}^{c} \in \mathcal{P}_{1}(c)$ follows immediately from Theorem 1 . To show $\underline{P}_{1}^{c} \in \mathcal{P}_{1}(c)$, we show that the transitive closure $\tilde{P}_{2}^{c}$ of $\succ_{D}^{c} \cup \bar{P}_{1}^{c} \backslash \underline{P}_{1}^{c}$ is asymmetric.

First, we define a $\tilde{P}_{2}^{c}$-cycle of length $n$ as a sequence $\left\{x_{i}\right\}_{i=0}^{n}$ that satisfies the following: (i) for all $i, x_{i}>_{D}^{c} x_{i+1}$, or $x_{i}^{c} \tilde{P}_{1}^{c} x_{i+1}$, where $\tilde{P}_{1}^{c} \equiv \bar{P}_{1}^{c} \backslash \underline{P}_{1}^{c}$; (ii) $x_{i} \neq x_{j}$ for all $i, j \neq 0, n$ with $i \neq j$; and (iii) $x_{n}=x_{0}$. Because of NBCC, we can assume $x_{0} \tilde{P}_{1}^{c} x_{1}$, and $x_{1}>_{D}^{c} x_{2}$ without loss of generality. To complete the proof, we only need to show that $\tilde{P}_{2}^{c}$-cycles do not exist for all $n \geq 3$. We proceed by induction.

Suppose $n=3$. Because $x_{0} \tilde{P}_{1}^{c} x_{1}$, we must have $x_{2}>_{D}^{c} x_{0}$. Otherwise, $x_{2}=c\left(\left\{x_{0}, x_{2}\right\}\right)$ implies $x_{2} \bar{P}_{1}^{c} x_{0}$, and then $x_{2} \bar{P}_{1}^{c} x_{1}$, a contradiction. Then together with $x_{1}>_{D}^{c} x_{2}$, we have 
$x_{1} \succ_{I}^{c} x_{0}$, which contradicts $x_{0} \tilde{P}_{1}^{c} x_{1}$. Therefore, $\tilde{P}_{2}^{c}$-cycles of length 3 do not exist.

Next, assume that there do not exist any $\tilde{P}_{2}^{c}$-cycles of length shorter than $m$. We need to show that $\tilde{P}_{2}^{c}$-cycle of length $m$ does not exist.

Observe first that $x_{0}=c\left(\left\{x_{0}, x_{2}\right\}\right)$ because of an argument similar to the case $n=3$. There are three possibilities: (i) $x_{0}>_{D}^{c} x_{2}$; (ii) $x_{0} \tilde{P}_{1}^{c} x_{2}$; or (iii) $x_{0} \underline{P}_{1}^{c} x_{2}$. If either (i) or (ii) holds, then $\left\{x_{0}, x_{2}, \cdots, x_{m}\right\}$ is a $\tilde{P}_{2}^{c}$-cycle of length $m-1$. This contradicts the induction hypothesis. Suppose (iii) holds. Then, by definition of $\underline{P}_{1}^{c}$, we have $x_{2}>_{I}^{c} x_{0}$. Together with $x_{1}>_{D}^{c} x_{2}$, we have $x_{1}>_{I}^{c} x_{0}$, by the definition of $>_{I}^{c}$. But $x_{1}>_{I}^{c} x_{0}$ contradicts $x_{0} \tilde{P}_{1}^{c} x_{1}$.

Let $\tilde{P}_{2}^{c \prime}$ be a completion of $\tilde{P}_{2}^{c}$, which exists by Szpilrajn's Theorem. Since $\underline{P}_{1}^{c}$ is transitive by construction, it is straightforward to see $\left(\underline{P}_{1}^{c}, \tilde{P}_{2}^{c \prime}\right) \in \mathcal{P}(c)$.

Proof of (ii): Let $\left(P_{1}^{c}, P_{2}^{c}\right) \in \mathcal{P}(c)$.

(ii.a) Recall that $x \bar{P}_{1}^{c} y$ if and only if $c(S)=c(S \backslash\{y\})$ for all $S \ni x, y$ and $x \neq y$. Therefore, $P_{1}^{c} \subset \bar{P}_{1}^{c}$.

Now we show $\underline{P}_{1}^{c} \subset P_{1}^{c}$. First note that $>_{D}^{c} \subset P_{2}^{c}$. This is because $x>_{D}^{c} y$ if and only if $x=c(\{x, y\})$, and $c(S) \neq c(S \backslash\{y\})$ for some $S \ni x, y$. If $x P_{1}^{c} y$, then $c(S)=c(S \backslash\{y\})$ for all $S \ni x, y$, and hence, $x>_{D}^{c} y$ implies $x P_{2}^{c} y$, i.e., $>_{D}^{c} \subset P_{2}^{c}$.

Suppose $x \underline{P}_{1} y$ but $\neg\left(x P_{1}^{c} y\right)$. Note $x \underline{P}_{1} y$ if and only if $x=c(\{x, y\})$ and there exists a sequence $\left\{z_{i}\right\}_{i=1}^{n}$ such that $y>_{D}^{c} z_{1}>_{D}^{c} \cdots>_{D}^{c} z_{n}>_{D}^{c} x$. Therefore, $x \underline{P}_{1} y$ implies $y P_{2}^{c} x$ for any $P_{2}^{c} \in \mathcal{P}_{2}(c)$. However, $\neg\left(x P_{1}^{c} y\right)$ and $x=c(\{x, y\})$ imply $x P_{2}^{c} y$, a contradiction.

(ii.b) Suppose $P_{1}^{c} \subset P_{1}^{c \prime}$, and $P_{1}^{c}, P_{1}^{c \prime} \in \mathcal{P}_{1}(c)$. The proof is complete if we show $\mathcal{P}_{2}\left(P_{1}^{c}, c\right) \subset \mathcal{P}_{2}\left(P_{1}^{c,}, c\right)$.

Pick some $P_{2}^{c} \in \mathcal{P}_{2}\left(P_{1}^{c}, c\right)$. It suffices to show that $\left(P_{1}^{c \prime}, P_{2}^{c}\right) \in \mathcal{P}(c)$. Fix a choice set $S$ and let $x=c(S)$. Then $x \in \max \left(S ; P_{1}^{c \prime}\right) \subset \max \left(S ; P_{1}^{c}\right)$. Given that $x P_{2}^{c} y$ for all $y \in \max \left(S ; P_{1}^{c}\right)$, we have $x P_{2}^{c} y$ for all $y \in \max \left(S ; P_{1}^{c \prime}\right)$. This implies $x=c_{\left(P_{1}^{c}, P_{2}^{c}\right)}(S)$.

$\left|\mathcal{P}_{2}\left(\underline{P}_{1}^{c} ; c\right)\right|=1$ follows from the construction of $\underline{P}_{1}$. Q.E.D. 


\section{References}

Aspesteguia, Jose and Angel Ballester, "Rationalizability of Choice by Sequential Procedures," 2009. Mimeo, Universitat Autonoma de Barcelona.

Cherepanov, Vadim, Timothy Feddersen, and Alvaro Sandroni, "Rationalization," 2008. Mimeo, Northwestern University.

García-Sanz, María D. and José Carlos R. Alcantud, "Rational choice by two sequential criteria," 2010. Mimeo, Universidad de Salamanca.

Kreps, David M., Notes on the Theory of Choice, Boulder: Westview Press, 1988.

Loomes, Graham, Chris Starmer, and Robert Sugden, “Observing Violations of Transitivity by Experimental Methods," Econometrica, March 1991, 59 (2), 425-39.

Luce, R. Duncan, "Semiorders and a Theory of Utility Discrimination," Econometrica, 1956, 24 (2), pp. 178-191.

Manzini, Paola and Marco Mariotti, "Sequentially Rationalizable Choice," American Economic Review, December 2007, 97 (5), 1824-1839.

_ and _ , "Choice by Lexicographic Semiorders," Theoretical Economics, 2010. Forthcoming.

Masatlioglu, Yusufcan, Daisuke Nakajima, and Erkut Ozbay, "Revealed Attention," 2010. Mimeo, University of Michigan.

Starmer, Chris and Robert Sugden, "Testing Alternative Explanations of Cyclical Choices," Economica, August 1998, 65 (259), 347-61.

Tversky, Amos, "Intransitivity of Preferences," Psychological Review, January 1972, 79, 281-99. 